\title{
PADRÕES ONTOGENÉTICOS DAS ESTERASES, LEUCINA AMINOPEPTIDASE E $\alpha$-GLICEROFOSFATO DESIDROGENASE EM Anopheles (Nyssorhynchus) albitarsis LYNCH-ARRIBÁLZAGA, 1878 (DIPTERA: CULICIDAE).
}

\author{
Juracy de Freitas MAIA ${ }^{1}$, Joselita Maria Mendes dos SANTOS ${ }^{1}$
}

RESUMO - Modificações na expressão gênica foram observadas nos sistemas esterase, leucina aminopeptidase e $\alpha$-glicerofosfato desidrogenase, durante o desenvolvimento ontogenético de Anopheles albitarsis. A esterase revelou quatro regiões de atividade, sendo a esterase 1 detectada apenas em larvas de $4^{\circ}$ estádio velhas e em pupas, as esterases 2 e 4 foram presentes durante todo o desenvolvimento, e a esterase 3 revelou-se praticamente apenas em larvas e raríssimas vezes em pupas. Também foram observadas quatro regiōes de atividade na leucina aminopeptidase, durante a ontogenia. As LAP1 e LAP2 foram características de larvas, a LAP3 esteve presente somente em pupas e adultos e a LAP4 foi detectada nos três diferentes estágios. Uma única região foi observada para a $\alpha$-glicerofosfato desidrogenase e a intensidade de sua atividade cresce à medida que se aproxima o estágio adulto.

Palavras-chave: Anopheles albitarsis, isoenzimas, ontogenia, malária, Amazônia.

Ontogenetic Patterns of Esterases, Leucine Aminopeptidase and $\alpha$-Glycerophosphate Dehydrogenase in Anopheles (Nyssorhynchus) albitarsis Lynch-Arribálzaga, 1878 (Diptera: Culicidae).

ABSTRACT - Modifications in genetic expression were observed in the esterase, leucine aminopeptidase and $\alpha$-glycerophosphate dehydrogenase systems during ontogenetic development of Anopheles albitarsis. The esterase revealed four regions of activity. Esterase 1 was detected in 4 th stage larvae and in pupae. Esterase 2 and 4 were present during all stages of development and esterase 3 appeared mainly in larvae and rarely in pupae. Four regions of activity were also observed in leucine aminopeptidase during ontogeny. The LAP1 and LAP2 were characteristic of larvae and LAP3 was present only in pupae and adults. LAP4 was detected in all three stages. $\alpha$-glycerophosphate dehydrogenase presented one activity band whose intensity increased with development.

Key-word: Anopheles albitarsis, isozymes, ontogeny, malaria, Amazon.

\section{INTRODUÇÃO}

\section{A distribuição de Anopheles} albitarsis é ampla na região neotropical, ocorrendo desde o nordeste da Argentina e estendendo-se por toda a América do Sul, com exceção da parte ocidental. Na América Central, tem sido registrada no Panamá, Costa Rica, Honduras e Guatemala (Galvão \& Damasceno, 1944; Forattini, 1962; Ferreira, 1964). Apresenta consideráveis variações morfológicas, bioquímicas e comportamentais, o que se reflete numa heterogeneidade no papel de transmissor da malária humana. Considerado como um vetor primário (Rachou, 1958; Ferreira, 1964), Deane (1986) menciona-o como um vetor de baixo poder de transmissão, mas com importância em áreas restritas.

As isoenzimas e sua análise através da eletroforese são consideradas ferramentas importantes em pesquisa básica e aplicada. Cada organismo possui seu próprio padrão eletroforético de enzimas, e este pode mudar durante o desenvolvimento, O estudo isoenzimático permite determinar a ação gênica

1 Instituto Nacional de Pesquisas da Amazônia - INPA. Cx. Postal 478, 69.011-970 Manaus, AM, Brasil. 
diferencial e o momento em que um gene específico entra em atividade na sintese da enzima correspondente (Wagner \& Selander, 1974). Mudanças nos padrões de isoenzimas são indicadoras de modificações na expressão dos genes e reflexos de eventos genéticos e epigenéticos intra e extra celulares (Whitt, 1975). Vários estudos em espécies de Anopheles tem mostrado que algumas isoenzimas modificam-se durante a ontogenia, seja em termos de intensidade das bandas ou no aparecimento de novas formas e o desaparecimento de outras (Pantelouris \& Downer, 1969; Vedbrat \& Whitt, 1974; Revanasiddaiah \& Chowdaiah, 1982; Santos et al., 1985; Scarpassa et al., 1992; Scarpassa \& Tadei, 1993; Santos et al., 1996).

Este trabalho, refere-se aos padrões eletroforéticos das isoenzimas de esterases, leucina aminopeptidase e $\alpha$-glicerofosfato desidrogenase, durante o desenvolvimento ontogenético de Anopheles albitarsis, com o objetivo de verificar modificações na expressão gênica e determinar possiveis atividades na síntese da enzima correspondente.

\section{MATERIAL E MÉTODOŚS}

O material utilizado foi procedente de Maruanum, aproximadamente 60 quilômetros a noroeste de Macapá $\left(0^{\circ}\right.$ $02^{\prime} 20^{\prime \prime} \mathrm{N}, 51^{\circ} 03^{\prime} 59^{\prime \prime} \mathrm{W}$ ), no Estado do Amapá. Os espécimes foram capturados em um curral de gado, nas condições de exofagia e zoofilia, com auxílio de um aspirador entomológico manual e lanterna, sendo as fêmeas separadas individualmente para oviposição e identificadas por meio das chaves de Gorham et al. (1967) e Consoli \& Lourenço-de-Oliveira (1994). Após a eclosão dos ovos, as larvas de cada desova foram mantidas em insectário a $26 \pm 1{ }^{\circ} \mathrm{C}$ e alimentadas até atingirem o estágio adulto, conforme descrito em Santos et al. (1981).

Larvas de $4^{\circ}$ estádio, pupas e adultos de cada desova foram acondicionados separadamente em tubos plásticos e mantidos em freezer a temperatura de $-70{ }^{\circ} \mathrm{C}$. Nas análises eletroforéticas, utilizou-se cinco indivíduos de cada estágio por postura, num total de 100 amostras por sistema enzimático. As larvas de $4^{\circ}$ estádio foram separadas em três fases: jovens (até 12 horas), médias (12-24 horas) e velhas pigmentadas (após 24 horas). Foi empregado o sistema horizontal em gel de amido parcialmente hidrolisado (Sigma) a $12 \%$. Na preparação das amostras para as análises, sistema tampões e soluções de coloração seguiu-se a metodologia empregada por Santos et al. (1996).

\section{RESULTADOS}

\section{Esterases}

A análise desta enzima durante desenvolvimento ontogenético, por meio de $\alpha$-naftil propionato, revelou quatro regiões de atividade muito complexas, todas eletronegativas e mudanças na intensidade das bandas de acordo com o estágio de desenvolvimento (Fig. 1).

Dessas, apenas a primeira região não apresentou variação. $O$ perfil eletroforético das isoenzimas polimórficas 


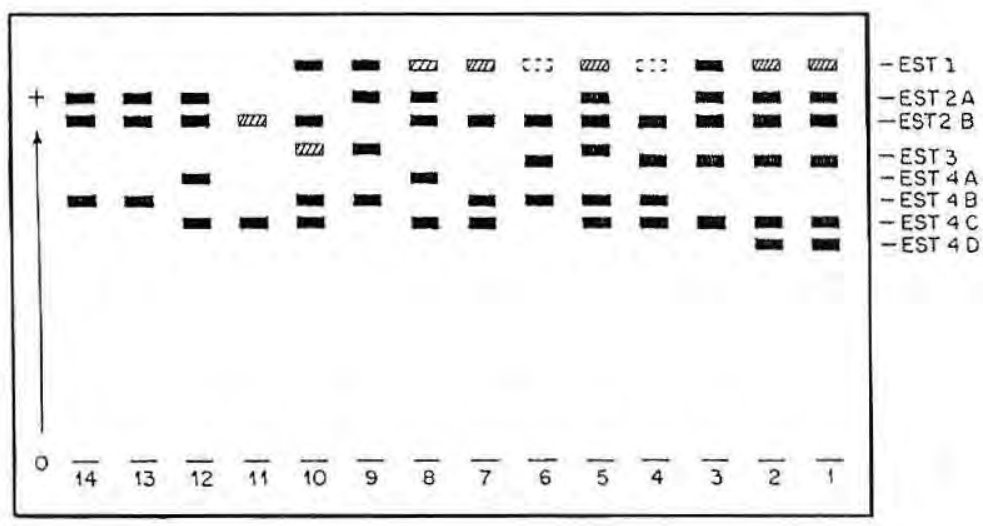

B

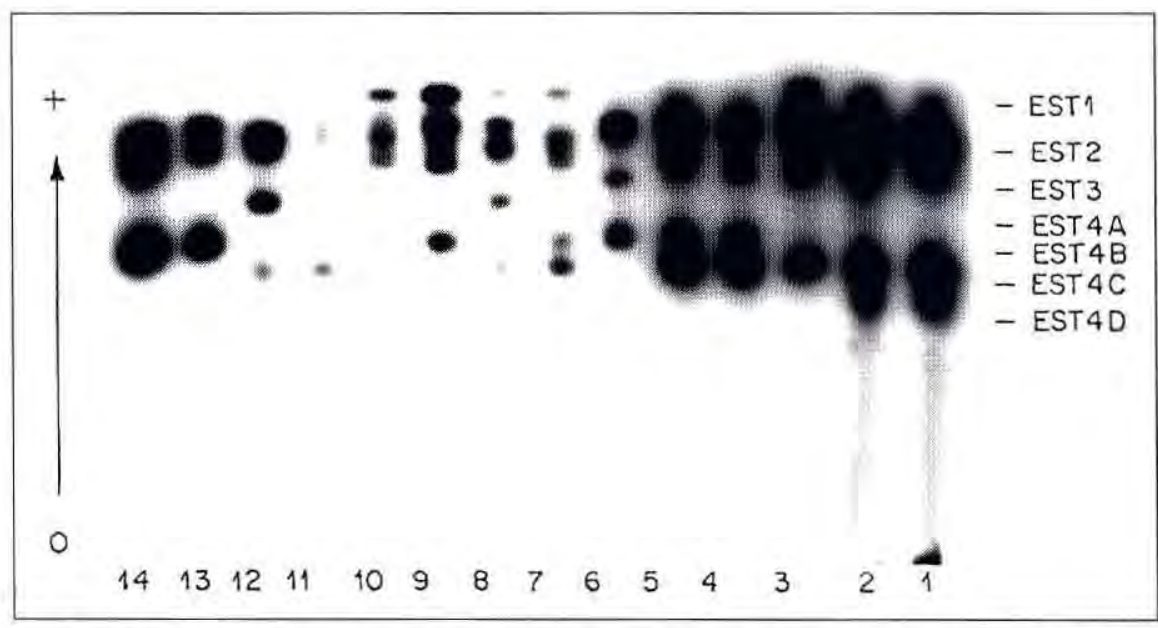

Figura 1. Padrões eletroforéticos das esterases, mostrando as quatro regiões de atividade durante o desenvolvimento ontogenético de Anopheles albitarsis. Eletroforese em gel de amido. Sistema tampão Tris-citrato-borato $\mathrm{pH}$ 8,0. Migração no sentido indicado. A - esquema e em $\mathbf{B}$ - fotografia. Amostras: 1 a $6=$ larvas de $4^{\circ}$ estádio, 7 a $10=$ pupas e 11 a $14=$ adultos. $=$ atividade forte; $=$ atividade média e $\square=$ atividade fraca. 
apresentaram três fenótipos distintos: uma banda de mobilidade rápida, outra lenta $\mathrm{e}$ o terceiro mostrando duas bandas de igual intensidade, sugerindo estrutura monomérica para as proteinas. O controle genético para os locos EST2 e EST3 foi determinado por dois alelos codominantes - EST2*A e $E S T 2 * B ; E S T 3 * A$ e $E S T 3 * B$, respectivamente. Enquanto que para o loco EST4 foram identificados quatro alelos ${ }_{\text {EST4* }}^{*} A, E S T 4^{*} B, E S T 4^{*} C$ e EST4*D.

A esterase 1 foi revelada em larvas de $4^{\circ}$ estádio, em pupas e totalmente ausente no estágio de adulto. Baixa intensidade de coloração das bandas foi observada nas primeiras horas da maioria das larvas de $4^{\circ}$ estádio, alcançando o máximo de atividade em larvas velhas (com pigmentação escura, ou seja, em fase de pré-pupa) e em pupas recém eclodidas, diminuindo a medida que estas últimas completavam seu ciclo e desaparecendo completamente nos adultos.

A esterase 2 mostrou-se presente nos três estágios de desenvolvimento, sendo que esta foi detectada com maior intensidade de coloração em larvas de $4^{\circ}$ estádio.

A esterase 3 foi observada algumas vezes em larvas de $4^{\circ}$ estádio, raríssimas vezes em pupas e nunca detectada em adultos.

A esterase 4 foi revelada em todos os estágios de desenvolvimento, apresentando bandas com maior intensidade de coloração em larvas de $4^{\circ}$ estádio.

\section{Leucina Aminopeptidase}

O padrão eletroforético da leucina aminopeptidase, durante o desenvolvimento ontogenético, mostrou quatro regiões de atividade, todas eletronegativas, e denominadas de LAP1 a LAP4 com modificações na expressão gênica conforme o estágio considerado (Fig. 2). Apenas a região LAP2 apresentou variação, controlada geneticamente por dois alelos codominantes $L A P 2 * A$ e $L A P 2 * B$, cujo perfil do fenótipo heterozigoto sugere que a proteina seja monomérica.

LAP1 e LAP2 foram características de larvas de $4^{\circ}$ estádio e mostraram forte intensidade de coloração sobre o gel. A LAP3 foi específica dos estágios de pupas e adultos, sendo que nas primeiras, as bandas foram coradas mais intensamente. Para a LAP4, o padrão obtido foi semelhante à LAP3, entretanto ela também foi detectada em larvas velhas, próximas ao estágio de pupa, e maior intensidade de coloração das bandas foi observada em adultos. Algumas vezes, apareceu uma região corada próxima à origem no estágio de larvas, a qual não foi considerada, atribuindo-se ser uma isoenzima secundária.

\section{$\alpha$-Glicerofosfato Desidrogenase}

O perfil eletroforético da $\alpha$ glicerofosfato desidrogenase, obtido durante o desenvolvimento, mostrou apenas uma região de atividade próxima à origem, também eletronegativa e monomórfica (Fig. 3). A intensidade de coloração das bandas foi fraca em larvas de $4^{\circ}$ estádio, aumentando em pupas e alcançando o máximo de atividade em adultos.

\section{DISCUSSÃO}

As quatro regiões de atividade esterásica, observadas durante o desenvolvimento de $A$. albitarsis, mostraram 
A

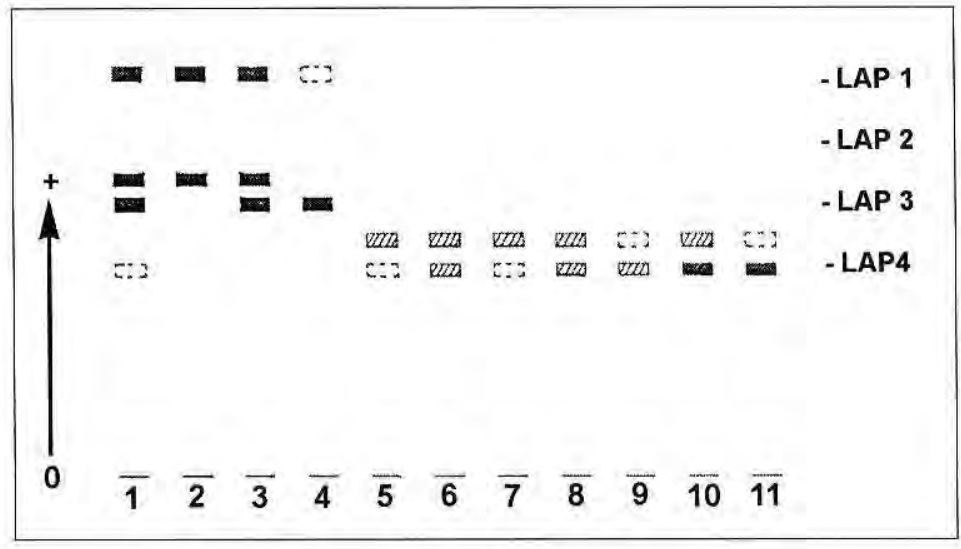

B

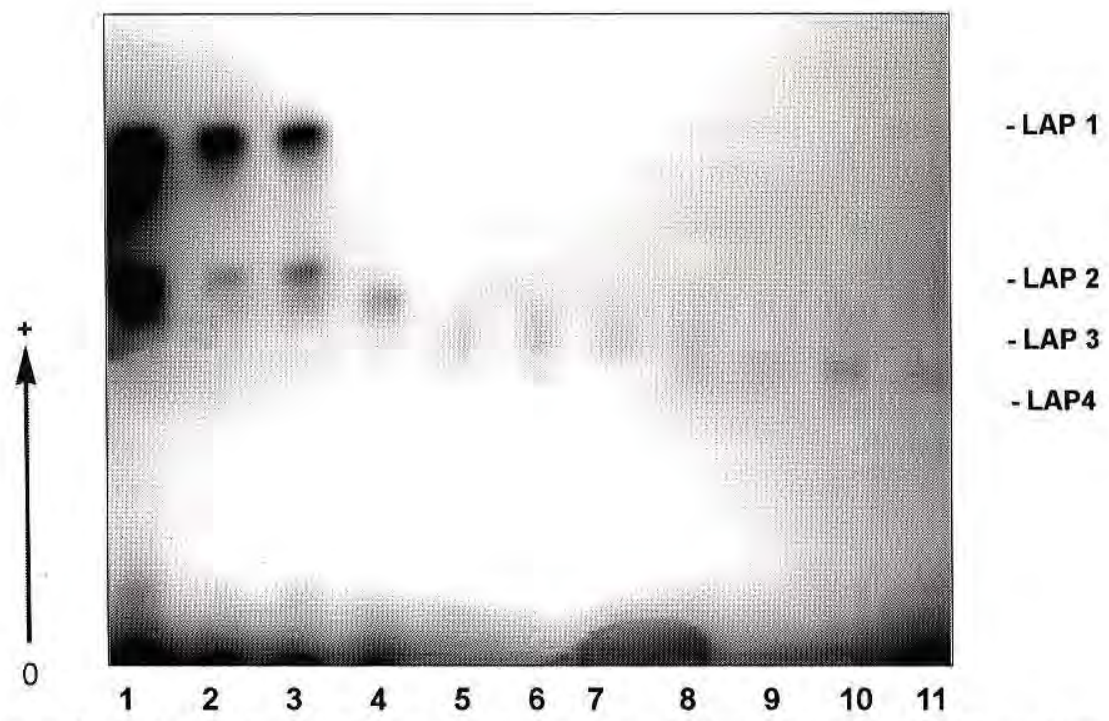

Figura 2. Padrões eletroforéticos das isoenzimas de leucina aminopeptidase durante o desenvolvimento ontogenético de Anopheles albitarsis. Eletroforese em gel de amido. Sistema tampão Tris-citrato-borato $\mathrm{pH} \mathrm{8,0.} \mathrm{Migração} \mathrm{no} \mathrm{sentido} \mathrm{indicado.} \mathrm{A} \mathrm{-} \mathrm{esquema} \mathrm{e} \mathrm{em} \mathbf{B}$ fotografia, mostrando as quatro zonas de atividade observadas. Amostras: 1 a $4=$ larvas de $4^{\circ}$ estádio, 5 a 8 = pupas e 9 a $11=$ adultos.

- = atividade forte; $=$ atividade média e $\square=$ atividade fraca 


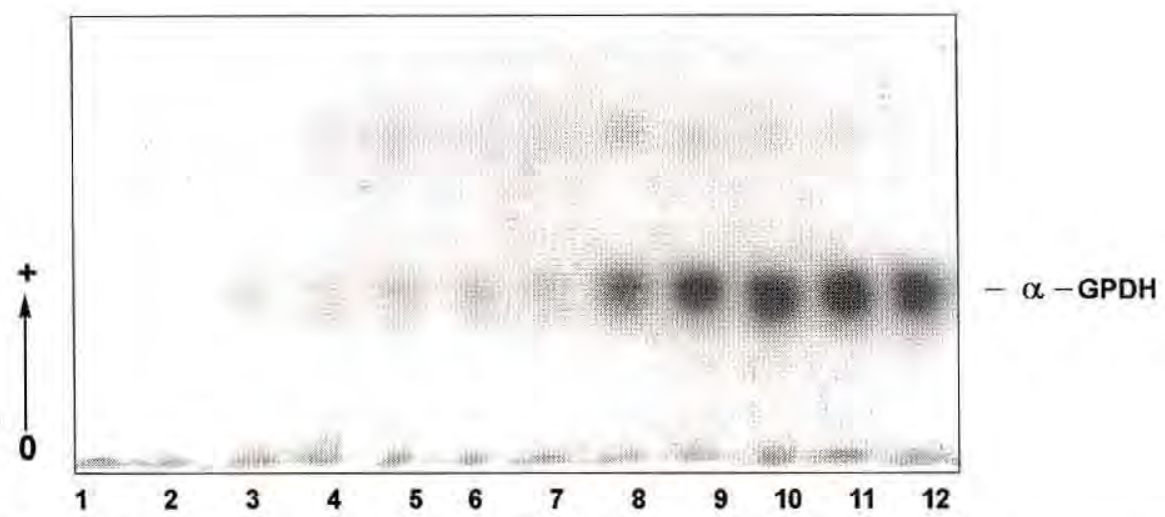

Figura 3. Perfil eletroforético da $\alpha$-glicerofosfato desidrogenase durante o desenvolvimento ontogenético de Anopheles albitarsis. Eletroforese em gel de amido. Sistema tampão Tris-fosfato pH 7,4. Migração no sentido indicado. Amostras: 1 a $3=$ larvas de $4^{\circ}$ estádio, 4 a $7=$ pupas e 8 a $12=$ adultos.

diferenças qualitativas e quantitativas e mudanças nos perfis eletroforéticos, quando comparamos larvas ( $4^{\circ}$ estádio), pupas e adultos.

Considerando-se os resultados obtidos, verifica-se que a esterase 1 foi registrada em larvas e pupas. As esterases 2 e 4 foram observadas em todos os estágios do desenvolvimento e a esterase 3 foi revelada em larvas de $4^{\circ}$ estádio, praticamente não foi detectada em pupas e totalmente ausente nos adultos.

Diferenças na expressão gênica durante o desenvolvimento, também foram verificadas em Anopheles albimanus, Anopheles nuneztovari e Anopheles darlingi. Na primeira, destas espécies, das nove regiões detectadas, a esterase 1 foi revelada em larvas e pupas, enquanto que as esterases 5 e 7 apenas em larvas, as esterases 3 e 9 foram específicas de pupas e as esterases 2, 4, 6 e 8 mostraram-se presentes em todos os estágios do desenvolvimento (Vedbrat \& Whitt, 1975).

Em A. nuneztovari, Scarpassa
(1988) observou sete regiões de atividade esterásica. As esterases 1 e 5 foram reveladas durante todo $o$ desenvolvimento, sendo que a esterase 1 apresentou forte intensidade de coloração e a esterase 5 fraca. As esterases 2 e 6 apresentaram forte intensidade nos estádios larvais e fraca nos estágios de pupas e adultos. A esterase 3 praticamente não foi registrada nos dois últimos estágios, denotando uma isoenzima característica do estágio larval. A esterase 4 mostrou-se mais corada nas larvas, diminuindo sua intensidade gradativamente no estágio de pupa e estando quase ausente nos adultos. A esterase 7 foi registrada nos primeiros estádios larvais, em larvas de $4^{\circ}$ estádio velhas e em frequência baixa em pupas e adultos.

Santos et al. (1996), analisando quatro populações de $A$. darlingi da região amazônica, constataram 5 regiões de atividade esterásica, durante o desenvolvimento dessa espécie. As esterases le 2 mostraram maior intensidade de coloração no estágio de larva e 
menos intensamente em pupas e adultos. $\mathrm{O}$ oposto ocorreu com as esterases $3 \mathrm{e}$ 4 , que foram melhor visualizadas nestes dois últimos estágios. A esterase 5 foi detectada em todos os estágios de desenvolvimento.

Considerando os dados da literatura, propôs-se hipóteses relacionadas às funções das quatro isoenzimas de esterases durante os diferentes estágios de $A$. albitarsis. Esterase 1, que mostra baixa atividade em larva de $4^{\circ}$ estádio velha e vai aumentando até pupas novas e desaparece em pupas velhas e adultos, pode estar relacionada à regulação dos niveis de ecdisona, admitindo-se que esta isoenzima no estágio de pupa desempenhe alguma função de regulação no metabolismo desse hormônio, como proposto por Vedbrat \& Whitt (1975) para a esterase 1 de $A$. albimanus e por Santos et al. (1985) para as esterases 1 e 2 de $A$. darlingi.

As esterases $2 \mathrm{e} 4$, presentes durante todos os estágios do desenvolvimento de A. albitarsis, poderiam estar relacionadas a funções mais generalizadas, supondo-se que estas possam desempenhar algum papel na quebra de lipídios, os quais seriam utilizados como fonte de energia. Esta proposição está de acordo com o descrito para as esterases 2, 4, 6 e 8 de A. albimanus (Vedbrat \& Whitt, 1975), para as esterases 1 e 5 de $A$. nuneztovari (Scarpassa, 1988) e a esterase 5 de $A$. darlingi (Santos, 1992). Com relação à esterase 3 , de $A$. albitarsis, detectada em larvas de $4^{\circ}$ estádio, pode-se supor que esteja associada com o metabolismo larval que é distinto do adulto. As larvas alimentando-se de microorganismos, alimentos triturados ou similares podem requerer esta esterase, enquanto que os adultos não a utilizariam, pois alimentam-se de soluções açucaradas. Hipótese semelhante foi proposta por Vedbrat \& Whitt (1975) para as esterases 5 e 7 de A. albimanus e por Scarpassa (1988) para a esterase 3 de $A$. nuneztovari.

A leucina aminopeptidase é uma enzima proteolítica e provavelmente desempenha função importante na degradação de proteínas durante o desenvolvimento. Segundo Johnson \& Sakai (1964), o estudo ontogenético desta enzima, em diferentes organismos, permite separá-las em dois grupos: (1) a enzima presente em todos os estágios do desenvolvimento e (2) a produção da enzima limitada a estágios específicos.

No estudo ontogenético da leucina aminopeptidase em $A$. albitarsis, foi observado quatro regiões de atividade e diferenças na expressão gênica, conforme o estágio considerado. LAP1 e LAP2 presentes apenas em larvas de $4^{\circ}$ estádio, LAP3 característica de pupas e adultos e a LAP4 detectada em todos os estágios de desenvolvimento.

Resultados semelhantes foram obtidos por Narang et al. (1979) em A. nuneztovari. Estes autores detectaram quatro regiões denominadas de LAP-1 a LAP-4. A LAP-1 observada em larvas e ausente ou em baixa atividade em pupas e adultos. A LAP-2 somente detectada em larvas, enquanto que as LAP-3 e LAP-4 foram registradas nos três estágios, porém com atividade bem reduzida em pupas e adultos. Uma região mais catódica em 
relação à LAP-2 foi observada em pupas e adultos, sendo denominada de LAP2-PA. Contudo, Scarpassa et al. (1992) estudando uma população de Tucuruí (PA) dessa mesma espécie, registraram seis regiōes denominadas de LAP1 a LAP6. As LAP1, LAP2, LAP4 e LAP5 detectadas em todos os estágios do desenvolvimento, enquanto que as LAP3 e LAP6 foram reveladas em pupas e adultos.

Diferenças na expressão gênica, conforme o estágio de desenvolvimento, também foram descritas por Santos et al. (1996) em A. darlingi. Os autores verificaram seis regiões de atividade para a espécie denominadas de LAP1 a LAP6. ALAP1 1 LAP6 foram reveladas em todos os estágios e a LAP3 detectada em pupas e adultos. LAP2 e LAP5 foram especificas de larvas, enquanto que a LAP4 foi observada apenas em pupa.

De conformidade com a literatura, pode-se supor que a LAP3 de $A$. albitarsis, especifica de pupas e adultos, possa desempenhar alguma função na histólise dos tecidos larvais, como proposto por Sakai et al. (1969) para a região LAP-D de Drosophila melanogaster. Estes autores sugeriram que a alta atividade da enzima, particularmente no estágio de pupa, é devida a intensa histólise dos tecidos larvais, ao mesmo tempo que ocorre a diferenciação dos tecidos do adulto. A mesma hipótese foi sugerida por Scarpassa et al. (1992) para as LAP3 e LAP6 de A. nuneztovari de Tucuruí e por Santos et al. (1996) para as LAP3 e LAP4 de $A$. darlingi.

A expressão gênica da $\alpha$-GPDH du- rante o desenvolvimento de $A$. albitarsis mostrou que a atividade desta enzima aumenta durante o ciclo de vida do inseto. Padrão similar tem sido observado em outras espécies de Anopheles, variando entretanto no número de locos.

Vedbrat \& Whitt (1974) descreveram três formas da $\alpha$-GPDH de intensidade de coloração idênticas em larvas de A. albimanus e, a mais anódica, mostrando aumento de atividade em pupas e adultos. Scarpassa \& Tadei (1993) observaram apenas uma região para $A$. nuneztovari, contudo, as bandas detectadas no estágio larval mostraram diferença de mobilidade, sendo mais catódica cerca de 1-2 milimetros em relação às bandas de pupas e adultos. Em $A$. darlingi, Santos et al. (1996) relatam diferenças no padrão da $\alpha$-GPDH, quanto ao tipo de suporte empregado. Uma única região foi verificada em gel de amido, enquanto em géis de poliacrilamida, foram observadas duas. Contudo, a intensidade de coloração das bandas, nos dois suportes, foi fraca em larvas de $4^{\circ}$ estádio após 24 horas de eclosão, aumentou em pupas e atingiu o máximo na fase adulta.

$O$ aumento da atividade da enzima à medida que o inseto atinge a fase adulta, provavelmente signifique que possa estar ocorrendo aumento na produção da $\alpha$-GPDH. Esta atividade mais intensa no estágio adulto, permite admitir que a enzima possa apresentar maior concentração nos músculos alares, exercendo funções relacionadas à atividade de vôo da espécie, como sugerido por Scarpassa \& Tadei (1993) e Santos et al. (1996). 


\section{Bibliografia Citada}

Consoli, R. A. G. B.; Lourenço-de-Oliveira, R. 1994. Principais mosquitos de importância sanitária no Brasil. Rio de Janeiro, Fiocruz. p. 83-84.

Deane, L. M. 1986. Malaria vectors in Brazil. Mem. Inst. Oswaldo Cruz, 8I(suppl.II): 5-14.

Ferreira, E. 1964. Distribuição geográfica dos anofelinos no Brasil e sua relação com o estudo atual da erradicação da malária. Rev. Bras. Malar. D. Trop., 16: 329-348.

Forattini, O. P. 1962. Anophelini. In: Entomologia Geral. São Paulo, Faculdade de Higiene e Saúde Pública. v. 1, p. 376-380.

Galvão, A. L. A.; Damasceno, R. G. 1944. Observações sobre anofelinos do complexo albitarsis (Diptera: Culicidae). An. Fac. Med. Univ. São Paulo, 20: 73-87.

Gorham, J. R.; Stojanovich, C. J.; Scott, H. G. 1967. Clave ilustrada para los anofelinos de Sudamerica Oriental. Communicable Disease Center, United States Public Health Service, Atlanta, 64 p.

Johnson, F. M.; Sakai, R. K. 1964. A leucine aminopeptidase polymorphism in Drosophila buskii. Nature, 203(4943): 373-374.

Narang, S.; Santos, J. M. M.; Garcia, J. C.; Cristakou, H. D.; Narang, N. 1979. Genética de populações de anofelinos IV. Estudos eletroforéticos das populações naturais de Anopheles nuneztovari e Anopheles darlingi. Correlação genética entre espécies. Acta Amazonica, 9(3): 529-542.

Pantelouris, E. M.; Downer, R. G. 1969. Phenotypic changes of the esterase pattern in insect metamorphosis. J. Insect Physiol., 15: 2357-2362.

Rachou, R. G. 1958. Anofelinos do Brasil: Comportamento das espécies vetoras de malária. Rev. Bras. Malar: D. Trop., 10: 145-181.

Revanasiddaiah, H. M.; Chowdaiah, B. N. 1982. Electrophoretic studies on protein changes during developmental stages of Anopheles stephensi. Indian J. Med.Res., 75: 40-44.

Sakai, R. K.; Ting, D. A.; Scandalios, J. G. 1969. Developmental genetic studies of ami- nopeptidase in Drosophila melanogaster. Molec. Gen. Genetics, 105: 24-29.

Santos, J. M. M. 1992. Variabilidade Genética em Populações Naturais de Anopheles (Nyssorhynchus) darlingi Root, 1926 (Diptera: Culicidae). Tese de Doutoramento. PPGBTRN, INPA/FUA, Manaus, AM. 150 p.

Santos, J. M. M.; Contel, E. P. B.; Kerr, W. E. 1981. Biologia de Anofelinos Amazônicos. I. Ciclo biológico, postura e estádios larvais de Anopheles darlingi Root, 1926 (Diptera: Culicidae) da Rodovia Manaus/Boa Vista. Acta Amazonica, 11(4): 789-797.

Santos, J. M. M.; Contel, E. P. B.; Kerr, W. E. 1985. Biology of amazonian mosquitoes. III. Esterases isozymes in Anopheles darlingi. Acta Amazonica, 15(1/2): 167-177.

Santos, J. M. M.; Tadei, W. P.; Contel, E. P. B. 1996. Biology of amazonian anopheline. XX. Ontogeny of esterases, leucine aminopeptidase and $\alpha$-glycerophosphate dehydrogenase in Anopheles (Nyssorhynchus) darlingi Root, 1926 (Diptera: Culicidae). Rev. Brasil. Biol., 56(3): 591-598.

Scarpassa, V. M. 1988. Estudo do ciclo biológico e de isoenzimas na ontogênese de Anopheles (Nyssorhynchus) nuneztovari Gabaldón, 1940 (Diptera: Culicidae). Dissertação de Mestrado.INPA/FUA, Manaus, AM. 172 p.

Scarpassa, V. M.; Tadei, W. P. 1993. Biology of Amazonian Anophelines. XIX. $\alpha$-Glycerophosphate Dehydrogenase in Anopheles nuneztovari (Diptera: Culicidae): Ontogeny and Genetic Variation. Rev. Bras. Genet., 16(2): 297-306.

Scarpassa, V. M.; Tadei, W. P; Contel, E. P. B. 1992. Biologia de Anofelinos Amazônicos. XV. Leucina aminopeptidase em Anopheles (Nyssorhynchus) nuneztovari: ontogenia e variação genética. Acta Amazonica, 22(2): 229-238.

Vedbrat, S. S.; Whitt, G. S. 1974. Lactate dehydrogenase and glycerol-3-phosphate dehydrogenase gene expression during ontogeny of the mosquito Anopheles albimanus. J. Exp. Zool., 187: 135-140.

Vedbrat, S. S.; Whitt, G. S. 1975. Isozyme ontogeny of the mosquito, Anopheles albimanus. In: 
Isozymes, III. Developmental biology. New York, Academic Press. p. 131-143.

Wagner, R. P.; Selander, R. K. 1974. Isozymes in insects and their significance. Ann. Rev. Entomol., 19: 117-138.

Whitt, G. S. 1975. Isozymes and developmental biology. In: Market, C. L. (ed.). Isozymes. New York, São Francisco, London, Academic Press, V. 3. 